\title{
Identification of key genes in non-small cell lung cancer by bioinformatics analysis
}

\author{
Li Zhang ${ }^{1}$, Rui Peng ${ }^{2}$, Yan Sun ${ }^{1}$, Jia Wang ${ }^{1}$, Xinyu Chong ${ }^{1}$, Zheng Zhang $^{\text {Corresp. } 1}$ \\ ${ }^{1}$ Department of Molecular Medicine and Cancer Research Center, Chongqing Medical University, Chongqing, China \\ 2 Department of Bioinformatics, Chongqing Medical University, Chongqing, China \\ Corresponding Author: Zheng Zhang \\ Email address: zhangzheng92@163.com
}

\begin{abstract}
Background. Non-small cell lung cancer (NSCLC) is one of the most common malignant tumors in the world, and it has become the leading cause of death of malignant tumors. However, its mechanisms are not fully clear. The aim of this study is to investigate the key genes and explore their potential mechanisms involving in NSCLC.

Methods. We downloaded gene expression profile GSE33532, GSE30219 and GSE19804 from the Gene Expression Omnibus (GEO) database and analyzed by using GEO2R. Gene Ontology and Kyoto Encyclopedia of Genes and Genomes were used for the functional and pathway enrichment analysis. We constructed the protein-protein interaction (PPI) network by STRING and visualized it by Cytoscape. Further, we performed module analysis and centrality analysis to find the potential key genes. Finally, we carried on survival analysis of key genes by GEPIA.
\end{abstract}

Results. In total, we obtained 685 DEGs. Moreover, GO analysis showed that they were mainly enriched in cell adhesion, proteinaceous extracellular region, heparin binding. KEGG pathway analysis revealed that transcriptional misregulation in cancer, ECM-receptor interaction, cell cycle and p53 signaling pathway were involved in. Furthermore, PPI network was constructed including 249 nodes and 1027 edges. Additionally, a significant module was found, which included 8 candidate genes with high centrality features. Further, among the 8 candidate genes, the survival of NSCLC patients with the 7 high expression genes were significantly worse, including CDK1, CCNB1, CCNA2, BIRC5, CCNB2, KIAA0101 and MELK. In summary, these identified genes should play an important role in NSCLC, which can provide new insight for NSCLC research. 
1 Identification of key genes in non-small cell lung cancer by bioinformatics analysis

2 Li Zhang ${ }^{1}$, Rui Peng ${ }^{2}$, Yan Sun ${ }^{1}$, Jia Wang ${ }^{1}$, Xinyu Chong ${ }^{1}$, Zheng Zhang ${ }^{1 *}$

3

$4{ }^{1}$ Department of Molecular Medicine and Cancer Research Center, Chongqing Medical

5 University, Chongqing, China

$6 \quad{ }^{2}$ Department of Bioinformatics, Chongqing Medical University, Chongqing, China

8 Corresponding Author:

9 Zheng Zhang ${ }^{1}$

10 No.1 Medical College Road, Chongqing, 400016, China.

11 E-mail: zhangzheng92@163.com

\section{Abstract}

\section{Background.}

Non-small cell lung cancer (NSCLC) is one of the most common malignant tumors in the world, and it has become the leading cause of death of malignant tumors. However, its mechanisms are not fully clear. The aim of this study is to investigate the key genes and explore their potential mechanisms involving in NSCLC.

\section{Methods.}

We downloaded gene expression profile GSE33532, GSE30219 and GSE19804 from the Gene Expression Omnibus (GEO) database and analyzed by using GEO2R. Gene Ontology and Kyoto Encyclopedia of Genes and Genomes were used for the functional and pathway enrichment analysis. We constructed the protein-protein interaction (PPI) network by STRING and visualized it by Cytoscape. Further, we performed module analysis and centrality analysis to find the potential key genes. Finally, we carried on survival analysis of key genes by GEPIA.

\section{Results.}

In total, we obtained 685 DEGs. Moreover, GO analysis showed that they were mainly enriched in cell adhesion, proteinaceous extracellular region, heparin binding. KEGG pathway analysis revealed that transcriptional misregulation in cancer, ECM-receptor interaction, cell cycle and p53 signaling pathway were involved in. Furthermore, PPI network was constructed including 249 nodes and 1027 edges. Additionally, a significant module was found, which included 8 candidate genes with high centrality features. Further, among the 8 candidate genes, the survival of NSCLC patients with the 7 high expression genes were significantly worse, including CDK1, CCNB1, CCNA2, BIRC5, CCNB2, KIAA0101 and MELK. In summary, these identified genes should play an important role in NSCLC, which can provide new insight for NSCLC research. 
Keywords: Non-small cell lung cancer, Bioinformatics analysis, Protein-protein interaction network, Centrality analysis, Survival analysis.

\section{Introduction}

Lung cancer is one of the most common malignant tumors in the world, and it has become the first cause of death in malignant tumors in urban population in China (Yang et al. 2018). Nonsmall cell lung cancer (NSCLC) as the main type of lung cancer accounts for about $80 \%$ of all cases compared with small cell carcinoma and about $75 \%$ of patients are already in the advanced stage (Boolell et al. 2015). Although prominent achievements have been made in early prognosis and treatment, the five-year survival rate of NSCLC is not optimistic $(30 \%-40 \%)$ (Spira \& Ettinger 2004). Thus, it is important to explore the molecular mechanisms of non small cell lung cancer to develop more effective treatment methods.

In recent years, a large number of bioinformatics have been applied to clinical research, and a number of disease-related data have also been produced (Kulasingam \& Diamandis 2008). It will provide more significant diagnosis and treatment of NSCLC and find more potential targets for more effective therapeutic strategies. As more and more high throughput sequencing technologies and microarray have been released, there have a wide range of medical oncology research. Recently, microarray technology was used to investigate genes expression profiling which have identified differentially expressed genes (DEGs) in tumor samples compared with non-tumor samples for the development and progression of NSCLC (Jin \& Liu 2016). However, the pathogenesis of NSCLC is inadequately understood.

In this study, we downloaded three original gene expression profile datasets from Gene Expression Omnibus, including GSE33532 (Li et al. 2018), GSE30219 (Miao et al. 2019) and GSE19804 (Ni et al. 2018). 685 differently expressed genes (DEGs) were identified between NSCLC tissues and the non-tumor tissues using GEO2R. In addition, the Gene Ontology (GO) functional annotation and Kyoto Encyclopedia of Genes and Genomes (KEGG) pathway analysis were performed using the DAVID database. A protein-protein interaction (PPI) network was constructed using Search Tool for the Retrieval of Interacting Genes (STRING). Moreover, module analysis and centrality analysis of the network were used to identify the potential core genes in NSCLC. Further, prognosis and survival analysis results showed seven key genes were related to the prognosis of NSCLC. The work will provide the help to search for the molecular targets for the diagnosis and treatment of NSCLC.

\section{Materials and Methods}


79

80

81

82

83

84

85

86

87

88

89

90

91

92

93

94

95 96

97

98

99

100

101

102

103

104

105

106

107

108

109

110

111

112

113

114

115

116

117

Microarray data.

Three gene expression profiles (GSE33532, GSE30219 and GSE19804) were obtained from the Gene Expression Omnibus database (http: //www.ncbi. nlm.nih. gov/geo/). All three datasets followed these criteria: (a) The samples consisted of two groups of NSCLC tissues and nontumor tissues; (b) The sample size of each study would be greater than 100; (c) They were updated recently (2018-2019); (d)The samples based on the same platform: GPL570. The array data of GSE33532 included 80 NSCLC interpatient (patient-to-patient) and intrapatient (tumor sub-sample) variations in stage I and II of NSCLC patients in Germany. GSE30219 consisted of 293 NSCLC tissue samples and 14 non-tumor samples in France. GSE19804 included 60 paired NSCLC and non-tumor tissue of non-smoking female lung cancer patients in Taiwan population (Lu et al. 2010).

\section{Identification of DEGs.}

The DEGs between NSCLC tissues and non-tumor tissues were identified by GEO2R (https://www.ncbi.nlm.nih.gov/geo/geo2r/), which is the Gene Expression Omnibus online tool to screen DEGs by comparing two different groups of samples under the same experimental condition (Piao et al. 2018). The adjusted P-value (q-value) was regarded as the standard to correct the occurrence of false-positive results using the Benjamini and Hochberg false discovery rate method. The cut-off criteria was $\mid \log _{2}$ fold-change $(\mathrm{FC}) \mid>1$ and $\mathrm{q}$-value $<0.01$. The intersections of DEGs from GSE33532, GSE30219 and GSE19804 were obtained by Venny 2.1.0 (http://bioinfogp.cnb.csic.es/tools/venny/) (Sun et al. 2018).

\section{GO Function and KEGG Pathway Enrichment Analysis.}

GO and KEGG is used to understand and simulate higher-order functional behaviors of cells or organisms from the genomic information (Hao et al. 2018). GO and KEGG annotation of DEGs were carried out through DAVID database (https://david.ncifcrf.gov/), which is an online program that can provide a comprehensive set of functional annotation tool (Huang da et al. 2009). The analysis of Gene Ontology involves three aspects: cell component, molecular function and biological process. Enrichment factor was considered as the cutoff criterion to indicate a statistically significant difference. And the top $25 \mathrm{GO}$ terms and the KEGG pathways were selected.

\section{Construction of PPI network and Module analysis of PPI network.}

STRING is online tool designed to evaluate the PPI information. To detect the potential 
118 relationship among those DEGs, we used STRING app in Cytoscape 119 (http://www.cytoscape.org/) and mapped the DEGs into STRING (Szklarczyk et al. 2019). The 120 combined score of $>0.4$ was used as the cut-off value in the STRING database to improve the

result (Shannon et al. 2003). The Cytoscape app Molecular Complex Detection (MCODE) was applied to create the modules in the PPI network (Bader \& Hogue 2003), and degree cut-off=2, node score cut-off $=0.2, \mathrm{k}$-core $=2$, and $\max$ depth $=100$ were regarded as the criteria. The pathway analysis of genes in each module was performed by DAVID. And GO and KEGG pathway analysis were also made to explore the potential information of the genes.

\section{Centrality analysis of PPI network.}

We search and predict the key genes of the network using the significant parameters of degree centrality, betweenness centrality, closeness centrality and eigenvector centrality (Ostovari et al. 2018). The four centrality scores of each vertex were calculated by Cytoscape. Degree, betweenness and closeness were calculated using Network Analyzer of Cytoscape (Assenov et al. 2008) and eigenvector was calculated using Cytoscape app CytoNCA (Tang et al. 2015). The score file for these four parameters was downloaded from the Cytoscape software, the $\mathrm{R}$ language was used to describe the distribution of the four parameters and calculate the correlation among the four key centralities.

\section{The effect of expressions of key genes on NSCLC patient survival.}

With the widespread use of chips and high-throughput sequencing, a large amount of genomics data has accumulated in the field of cancer research, such as TCGA (The cancer Genome Atlas) and ICGC (International Cancer Genome Consortium). Currently, GEPIA (http://gepia.cancerpku.cn/) is an interactive web server for cancer expression profile data containing 9736 tumor samples and 8587 normal samples from TCGA (The cancer Genome Atlas) and GTEx (Genotype-Tissue Expression), which provides customizable functions such as tumor and normal differential expression analysis, and we can get the expression of hub genes in NSCLC tissues and normal ones. $\mathrm{P}<0.01$ was selected as a threshold (Tang et al. 2017).

\section{Result}

\section{Identification of DEGs.}

In total, we obtained 14373, 21740 and 20748 DEGs from GSE33532, GSE30219 and GSE19804 datasets, 14078 genes were found to be existed in three databases (Figure 1A and Supplementary Table.1). Among these genes, we obtained 685 DEGs including 519 were upregulated and 166 were down-regulated genes between NSCLC tissues and non-tumor tissues $(|\log \mathrm{FC}|>1, \mathrm{P}<0.01)$ (Figure 1B). 
158

159

160

161

162

163

164

165

166

167

168

169

170

171

172

173

174

175

176

177

178

179

180

181

182

183

184

185

186

187

188

189

190

191

192

193

194

195

196

197

198

\section{Go and KEGG pathway enrichment analysis.}

The online tool of DAVID was used to analyze the GO and KEGG pathway enrichment based upon DEGs. The result of GO showed that the genes were involved in protein binding, integral component of membrane, integral component of plasma membrane, extracellular exosome, extracellular region, extracellular space of the cell component and positive regulation of transcription from RNA polymerase II promoter, cell adhesion, identical protein binding of the molecular function (Figure 2A). Moreover, the result of KEGG showed that the genes were mainly enriched in transcriptional misregulation in cancer, ECM-receptor interaction, cell cycle and p53 signaling pathway (Figure 2B).

\section{Construction of the NSCLC related PPI network.}

The PPI network of 685 DEGs was constructed from STRING to predict the interactions of identified DEGs, consisting of 249 nodes and 1027 edges, as shown in Supplementary Figure 1. The majority of the nodes in the PPI network were the upregulated DEGs in NSCLC samples. The PPI network mainly showed that the interactions between the molecules.

\section{Module analysis of the NSCLC related network.}

To explore the significance of the molecules of the NSCLC related PPI network, the Module analysis was performed by MCODE. Our result showed that there were eight modules in the PPI network by module analysis. A significant module with score $>2$ was found in Supplementary Figure 2. The module 1 was the most significant in whole modules, and it was located in the center of the entire PPI network. Moreover, the first-ranked module had the most nodes (33 nodes such as CDK1, CCNB1, CCNB2, CCNA2, KIAA0101, MELK and so on) and edges (510 edges). Furthermore, the genes were related to some enriched GO terms and pathways such as protein-binding, mitotic nuclear division, cell division, and so on (Supplementary Table.2).

\section{Centrality analysis of PPI network.}

To explore the features of the molecules of the NSCLC related PPI network, the centrality analysis was carried on by Cytoscape. Several parameters are used as centrality parameters in the centrality analysis, including degree, betweenness, closeness, eigenvector, information, subgraph, and so on. Among these, degree, betweenness, closeness and eigenvector are established important parameters in the centrality analysis. Degree centrality is the most direct measure of node centrality in network analysis. Betweenness centrality is an indicator of the importance of a node by the number of shortest paths passing through a node. Closeness centrality reflects the proximity of a node to other nodes in the network. Eigenvector centrality has the property that a 
199

200

201

202

203

204

205

206

207

208

209

210

211

212

213

214

215

216

217

218

219

220

221

222

223

224

225

226

227

228

229

230

231

232

233

234

235

236

237

238

239

vertex has a high score if it is connected with many other vertices with high scores. So, four topological features of the network centrality (degree, betweenness, closeness and eigenvector) were analyzed in our study. Our results showed that the distributions of degree, betweenness and eigenvector displayed the power-law distributions, while the distribution of closeness displayed a heavy-tailed distribution (Figure 3A-D). Moreover, the top 25\% of each parameter were chosen for further study (Table 1). Furthermore, 16 key DEGs with high topological features were obtained with high degree, betweenness, closeness and eigenvector (Figure 3E). Specifically, we found that eight among the 16 key DEGs were also in the module 1, including cyclin dependent kinase 1 (CDK1), cyclin B1 (CCNB1), cyclin A2 (CCNA2), baculoviral IAP repeat containing 5 (BIRC5), cyclin B2 (CCNB2), PCNA clamp associated factor (KIAA0101), maternal embryonic leucine zipper kinase (MELK) and enhancer of zeste 2 polycomb repressive complex 2 subunit $(\mathrm{EZH} 2)$.

\section{Survival analysis of key genes in NSCLC.}

GEPIA was applied to catch the 8 hub genes expression level between NSCLC tissues and normal ones. Our results showed that the seven high expressions of CDK1, CCNB1, CCNA2, BIRC5, CCNB2, KIAA0101 and MELK were associated with worse OS for NSCLC patients $(\mathrm{p}<0.01)$ (Figure 4A-G).

\section{Discussion}

The occurrence and development of lung cancer is a complex process because it involves aberrations of multiple genes and cellular pathways. It is vital important to find these hub genes and understand their roles in the molecular mechanism of NSCLC to improve the diagnosis and treatment (Liang et al. 2016). With the development of microarray and high-throughput technologies, identification of disease-associated genes and genes function prediction were applied.

In this study, based on three expression profiles of GSE33532, GSE30219 and GSE19804, 685 DEGs were identified, consisting of 519 upregulated and 166 downregulated genes between NSCLC tissues and non-tumor tissues. Moreover, GO results showed that the genes were involved in cell adhesion, extracellular region, integrin binding. Also, the result of KEGG showed that the genes were mainly enriched in transcriptional misregulation in cancer, ECMreceptor interaction, cell cycle and p53 signaling pathway. As known, the expression of cell adhesion molecules could affect the adhesive and signal transduction status of cells, and intimately related to the processes of cell motility and cell migration (Han et al. 2017). Also, researchers had found that integrin binding participated in variety of cellular functions including cell proliferation, survival and differentiation (Pan et al. 2016). In addition, cell cycle and cell proliferation were established terms related to tumor proliferation and apoptosis in lung cancer (An et al. 2016). Previous studies indicated that ECM-receptor interaction involved in the cell 
240 adhesion (Lessey \& Young. 1997), and ECM played an important role in tissue and organ 241 morphogenesis and in the maintenance of cell and tissue structure and function (Zakaria et al. 242 2015). Moreover, according to previous studies, this cisplatin exerted its cytotoxic effect which 243 associated with the p53 signaling pathway, and the disturbances in the p53 signaling pathway 244 were associated with NSCLC (Tang et al. 2018). Therefore, the data suggest that the identified DEGs may play roles in the occurrence and development of NSCLC.

246

247

248

249

250

251

252

253

254

255

256

257

258

259

260

261

262

263

264

265

266

267

268

269

270

271

272

273

274

275

276

277

278

279

280

To explore the molecular mechanism of NSCLC, the NSCLC related PPI network was constructed. Moreover, Module analysis showed that eight functional modules were detected. Specifically, the first-ranked module was found including 33 nodes and 510 edges. Previous studies have showed that modules analysis have been increasing used for identified the hub genes for various cancers liking hepatocellular carcinoma (Zhu et al. 2018), gastric cancer (Cao et al. 2018) and small cell lung cancer (Wen et al. 2018).Thus, these suggest that the nodes and edges in the first-ranked module may be the significant molecules of the network. Additionally, the results of centrality analysis showed that the distributions of degree, betweenness, closeness and eigenvector were typical form of heavy-tailed or close to typical form of heavy-tailed. As known, the typical forms of heavy-tailed were frequently found in many bio-molecular networks (Wang et al. 2014). Therefore, it suggests that the constructed networks have some similar statistical properties as the evolving network. Further, our data found 16 candidate molecules displayed high degree, betweenness, closeness and eigenvector. Finally, we found eight among 16 candidate molecules were consisted of the module 1 . Therefore, these results demonstrated that these 8 candidate molecules may be the important molecules in the NSCLC related PPI network and participant in NSCLC.

The survival and prognosis are important analytical indexes for evaluating disease prognosis, especially in cancer research. Our results showed that among the 8 candidate genes, the survival and prognosis of NSCLC patients with the seven high expression genes of CDK1, CCNB1, CCNA2, BIRC5, CCNB2, KIAA0101 and MELK were significantly worse $(p<0.01)$. CDK1, a cyclin-dependent kinase, is the center of the cell cycle control system and plays a very important role in the cell cycle progression (Ge et al. 2019). Studies had shown that CDK1 expression was associated with prognosis in advanced non-small cell lung cancer, and low expression of CDK1 had a better prognosis (Shi et al. 2017; Shi et al. 2016)). BIRC5 is a new member of the apoptosis inhibitory protein family and is the most potent inhibitor of apoptosis. BIRC5 has complex biological functions, inhibits apoptosis, promotes cell transformation and participates in cell mitosis, angiogenesis and drug resistance (Ebrahimiyan et al. 2019). It was reported that the expression level of BIRC5 was significantly higher in NSCLC tissues compared to non-tumor tissues, and negatively correlated with Tp53 (Wang et al. 2018; N. A. Vayshlya et al. 2008). CCNB2, a member of the cyclin family proteins, played an important role in the progression of G2/M transition by activating CDK1 kinase, and CCNB2 inhibition induced cell cycle arrest (Gao et al. 2019; Qian et al. 2015). CCNB1 was found to be associated with tumor aggressiveness and poor survival in lung cancer and esophageal cancer (Gao \& Wang 2018; 
281

282

283

284

285

286

287

288

289

290

291

292

293

294

295

296

297

298

299

300

301

302

303

304

305

306

307

308

309

310

311

312

313

314

315

316

317

318

319

320

321

Soria et al. 2000). The protein encoded by CCNA2 genes belongs to the highly conserved cyclin family, whose members function as regulators of the cell cycle (Krautgasser et al. 2019). However, the results about the function of CCNA2 on the prognosis in NSCLC were mutually contradictory. Some found that the expression of CCNA2 was correlated with prognosis, but others suggested that CCNA2 was not associated with prognosis of NSCLC (Xiao et al. 2018; Mengwei et al.2018). Therefore, the exact function of CCNA2 on prognosis of NSCLC needs further study. KIAA0101 was found to play an important role in the regulation of the cell cycle of NSCLC and the studies have showed that high-level KIAA0101 expression was also identified as an independent prognosis factor in NSCLC (Li et al. 2010; Kato et al. 2012). MELK, a cell cycle-dependent protein kinase, belongs to the KIN1/PAR-1/MARK family. MELK involved in the process of cell cycle, cell proliferation, tumor formation and apoptosis (Zhang et al. 2017). Moreover, MELK expression was elevated and closely related to the prognosis of NSCLC patients (Mullapudi et al. 2015; Christopher J Giuliano et al. 2018). Combined these together, It suggests that these seven genes have a close relation with the prognosis of NSCLC patients, and they may act as the prognostic indicators in NSCLC.

\section{Conclusions}

In conclusion, using multiple profile datasets and integrated bioinformatical analysis, we have identified 685 DEGs candidate genes at screening step, which significant enriched in several pathways, mainly associated with cell cycle and p53 signaling pathway. Then we filtered 249 nodes and 1027 edges in DEGs protein-protein interaction network complex, and found some candidate genes with high centrality features in a significant module. Finally, 7 key genes were found to be related to the prognosis of NSCLC patients, including CDK1, CCNB1, CCNA2, BIRC5, CCNB2, KIAA0101 and MELK. These findings could significantly improve our understanding of the cause and underlying molecular events in NSCLC, these candidate genes and pathways could be therapeutic targets for NSCLC. Combined these together, It suggests that these seven genes have a close relation with the prognosis of NSCLC patients, and they may act as the prognostic indicators in NSCLC. However, the functions and exact mechanisms of these seven genes in the prognosis of NSCLC, cell proliferation, cell migration, and so on need to be further studied.

\section{Acknowledgments}

This study was supported by Ying Yao Scientific Project of basic medicine college of Chongqing Medical University, China (JCYY201807).

\section{References}

An Q, Han C, Zhou Y, Li F, Li D, Zhang X, Yu Z, Duan Z, and Kan Q. 2016. Matrine induces 
322 cell cycle arrest and apoptosis with recovery of the expression of miR-126 in the A549 non-small

323

324

325

326

327

328

329

330

331

332

333

334

335

336

337

338

339

340

341

342

343

344

345

346

347

348

349

350

351

352

353

354

355

356

357

358

359

360

361

362

cell lung cancer cell line. Mol Med Rep 14:4042-4048. doi:10.3892/mmr.2016.5753.

Assenov Y, Ramirez F, Schelhorn SE, Lengauer T, and Albrecht M. 2008. Computing topological parameters of biological networks. Bioinformatics. 24: 282-284. doi:10.1093/bioinformatics/btm554.

Bader GD, and Hogue CW. 2003. An automated method for finding molecular complexes in large protein interaction networks. BMC Bioinformatics. 4:2.

Boolell V, Alamgeer M, Watkins DN, and Ganju V. 2015. The Evolution of Therapies in NonSmall Cell Lung Cancer. Cancers (Basel). 7:1815-1846. doi:10.3390/cancers7030864.

Cao L, Chen Y, Zhang M, Xu DQ, Liu Y, Liu T, Liu SX, and Wang P. 2018. Identification of hub genes and potential molecular mechanisms in gastric cancer by integrated bioinformatics analysis. PeerJ. 6:e5180. doi:10.7717/peerj.5180.

Christopher J Giuliano, Ann Lin, Joan C Smith, Ann C Palladino, Jason M Sheltzer. 2018. MELK expression correlates with tumor mitotic activity but is not required for cancer growth. eLife. 7:e32838.

Ebrahimiyan H, Rezaei N, Vojdanian M, and Aslani S. 2019. microRNA involvement in the regulation of survivin in peripheral blood mononuclear cells from rheumatoid arthritis patients. Int J Rheum Dis. 22(6):1107-1114. doi: 10.1111/1756-185x.13520.

Gao LW, and Wang GL. 2018. Comprehensive bioinformatics analysis identifies several potential diagnostic markers and potential roles of cyclin family members in lung adenocarcinoma. Onco Targets Ther. 11:7407-7415. doi:10.2147/ott.s171705.

Gao Z, Zhang D, Duan Y, Yan L, Fan Y, Fang Z, and Liu Z. 2019. A five-gene signature predicts overall survival of patients with papillary renal cell carcinoma. PLoS One. 14: e0211491. doi:10.1371/journal.pone.0211491.

Ge Y, He Z, Xiang Y, Wang D, Yang Y, Qiu J, and Zhou Y. 2019. The identification of key genes in nasopharyngeal carcinoma by bioinformatics analysis of high-throughput data. Mol Biol Rep. 46(3):2829-2840. doi:10.1007/s11033-019-04729-3.

Han L, Wang W, Ding W, and Zhang L. 2017. MiR-9 is involved in TGF-beta1-induced lung cancer cell invasion and adhesion by targeting SOX7. J Cell Mol Med 21:2000-2008. doi:10.1111/jcmm.13120.

Hao S, Li S, Wang J, Zhao L, Yan Y, Cao Q, Wu T, Liu L, and Wang C. 2018. Transcriptome Analysis of Phycocyanin-Mediated Inhibitory Functions on Non-Small Cell Lung Cancer A549 Cell Growth. Mar Drugs 16(12): E511. doi:16. 10.3390/md16120511.

Huang da W, Sherman BT, and Lempicki RA. 2009. Systematic and integrative analysis of large gene lists using DAVID bioinformatics resources. Nat Protoc. 4: 44-57. doi:10.1038/nprot.2008.211.

Jin X, Liu X, Li X and Guan Y. 2016. Integrated Analysis of DNA Methylation and mRNA Expression Profiles Data to Identify Key Genes in Lung Adenocarcinoma. Biomed Res Int. 2016:4369431. doi:10.1155/2016/4369431.

Kato T, Daigo Y, Aragaki M, Ishikawa K, Sato M, and Kaji M. 2012. Overexpression of KIAA0101 predicts poor prognosis in primary lung cancer patients. Lung Cancer. 75:110-118.

Peer] reviewing PDF | (2019:05:37984:1:1:NEW 1 Nov 2019) 
363

364

365

366

367

368

369

370

371

372

373

374

375

376

377

378

379

380

381

382

383

384

385

386

387

388

389

390

391

392

393

394

395

396

397

398

399

400

401

402

403

doi:10.1016/j.lungcan.2011.05.024.

Krautgasser C, Mandl M, Hatzmann FM, Waldegger P, Mattesich M, and Zwerschke W. 2019. Reliable reference genes for expression analysis of proliferating and adipogenically differentiating human adipose stromal cells. Cell Mol Biol Lett. 24:14. doi:10.1186/s11658-0190140-6.

Kulasingam V, and Diamandis EP. 2008. Strategies for discovering novel cancer biomarkers through utilization of emerging technologies. Nat Clin Pract Oncol 5:588-599. 10.1038/ncponc1187.

Lessey BA, and Young SL. 1997. Integrins and other cell adhesion molecules in endometrium and endometriosis. Semin Reprod Endocrinol. 15:291-299. doi:10.1055/s-2008-1068759.

Li H, Ma WL, Zuo CQ, Liang S, Ye Y, and Zheng WL. 2010. Functional prediction of KIAA0101 gene in human non-small cell lung cancer. Nan Fang Yi Ke Da Xue Xue Bao. 30:157-159.

Li S, Xuan Y, Gao B, Sun X, Miao S, Lu T, Wang Y, and Jiao W. 2018. Identification of an eight-gene prognostic signature for lung adenocarcinoma. Cancer Manag Res. 10: 3383-3392. doi:10.2147/cmar.s173941.

Liang B, Li C, and Zhao J. 2016. Identification of key pathways and genes in colorectal cancer using bioinformatics analysis. Med Oncol. 33:111. doi:10.1007/s12032-016-0829-6.

Lu TP, Tsai MH, Lee JM, Hsu CP, Chen PC, Lin CW, Shih JY, Yang PC, Hsiao CK, Lai LC, and Chuang EY. 2010. Identification of a novel biomarker, SEMA5A, for non-small cell lung carcinoma in nonsmoking women. Cancer Epidemiol Biomarkers Prev. 19: 2590-2597. doi:10.1158/1055-9965.epi-10-0332.

Mengwei Ni, Xinkui Liu, Jiarui Wu, Dan Zhang, Jinhui Tian, Ting Wang, Shuyu Liu, Ziqi Meng, Kaihuan Wang, Xiaojiao Duan, Wei Zhou and Xiaomeng Zhang. 2008. Identification of Candidate Biomarkers Correlated With the Pathogenesis and Prognosis of Non-small Cell Lung Cancer via Integrated Bioinformatics Analysis. Front Genet. 9: 469 . doi: 10.3389/fgene.2018.00469.

Miao R, Ge C, Zhang X, He Y, Ma X, Xiang X, Gu J, Fu Y, Qu K, Liu C, Wu Q, and Lin T. 2019. Combined eight-long noncoding RNA signature: a new risk score predicting prognosis in elderly non-small cell lung cancer patients. Aging (Albany NY). 11: 467-479. doi:10.18632/aging.101752.

Mullapudi N, Ye B, Suzuki M, Fazzari M, Han W, Shi MK, Marquardt G, Lin J, Wang T, Keller S, Zhu C, Locker JD, and Spivack SD. 2015. Genome Wide Methylome Alterations in Lung Cancer. PLoS One 10: e0143826. doi:10.1371/journal.pone.0143826.

N. A. Vayshlya ,M. V. Zinovyeva, A. V. Sass, E. P. Kopantzev, T. V. Vinogradova, E. D. Sverdlov. 2008. Increased expression of BIRC5 in non-small cell lung cancer and esophageal squamous cell carcinoma does not correlate with the expression of its inhibitors SMAC/DIABLO and PML. Cell Molecular Biology. Volume 42, Issue 4, pp 579-587.

Ni M, Liu X, Wu J, Zhang D, Tian J, Wang T, Liu S, Meng Z, Wang K, Duan X, Zhou W, and Zhang X. 2018. Identification of Candidate Biomarkers Correlated With the Pathogenesis and Prognosis of Non-small Cell Lung Cancer via Integrated Bioinformatics Analysis. Front Genet 9: 
404

405

406

407

408

409

410

411

412

413

414

415

416

417

418

419

420

421

422

423

424

425

426

427

428

429

430

431

432

433

434

435

436

437

438

439

440

441

442

443

444

469. 10.3389/fgene.2018.00469.

Ostovari M, Yu D, and Steele-Morris CJ. 2018. Identifying Key Players in the Care Process of Patients with Diabetes Using Social Network Analysis and Administrative Data. AMIA Annu Symp Proc 2018:1435-1441.

Pan J, Yang Q, Shao J, Zhang L, Ma J, Wang Y, Jiang BH, Leng J, and Bai X. 2016. Cyclooxygenase-2 induced beta1-integrin expression in NSCLC and promoted cell invasion via the EP1/MAPK/E2F-1/FoxC2 signal pathway. Sci Rep 6: 33823. doi:10.1038/srep33823.

Qian X, Song X, He Y, Yang Z, Sun T, Wang J, Zhu G, Xing W, and You C. 2015. CCNB2 overexpression is a poor prognostic biomarker in Chinese NSCLC patients. Biomed Pharmacother. 74: 222-227. doi:10.1016/j.biopha.2015.08.004.

Shannon P, Markiel A, Ozier O, Baliga NS, Wang JT, Ramage D, Amin N, Schwikowski B, and Ideker T. 2003. Cytoscape: a software environment for integrated models of biomolecular interaction networks. Genome Res. 13: 2498-2504. doi:10.1101/gr.1239303.

Sheng M, Dong Z, and Xie Y. 2018. Identification of tumor-educated platelet biomarkers of nonsmall-cell lung cancer. Onco Targets Ther. 11: 8143-8151. doi:10.2147/ott.s177384.

Shi Q, Zhou Z, Ye N, Chen Q, Zheng X, and Fang M. 2017. MiR-181a inhibits non-small cell lung cancer cell proliferation by targeting CDK1. Cancer Biomark. 20: 539-546. doi:10.3233/cbm-170350.

Shi YX, Zhu T, Zou T, Zhuo W, Chen YX, Huang MS, Zheng W, Wang CJ, Li X, Mao XY, Zhang W, Zhou HH, Yin JY, and Liu ZQ. 2016. Prognostic and predictive values of CDK1 and MAD2L1 in lung adenocarcinoma. Oncotarget. 7: 85235-85243. doi:10.18632/oncotarget.13252. Shukuya T, Yamada T, Koenig MJ, Xu J, Okimoto T, Li F, Amann JM, and Carbone DP. 2019. The Effect of LKB1 Activity on the Sensitivity to PI3K/mTOR Inhibition in Non-Small Cell Lung Cancer. J Thorac Oncol. 14: 1061-1076. doi:10.1016/j.jtho.2019.02.019.

Soria JC, Jang SJ, Khuri FR, Hassan K, Liu D, Hong WK, and Mao L. 2000. Overexpression of cyclin B1 in early-stage non-small cell lung cancer and its clinical implication. Cancer Res. 60: 4000-4004.

Spira A, and Ettinger DS. 2004. Multidisciplinary management of lung cancer. N Engl J Med. 350:379-392. doi:10.1056/NEJMra035536.

Sun S, Wang Y, Wu Y, Gao Y, Li Q, Abdulrahman AA, Liu XF, Ji GQ, Gao J, Li L, Wan FP, Li YQ, and Gao DS. 2018. Identification of COL1A1 as an invasionrelated gene in malignant astrocytoma. Int J Oncol. 53:2542-2554. doi:10.3892/ijo.2018.4568.

Szklarczyk D, Gable AL, Lyon D, Junge A, Wyder S, Huerta-Cepas J, Simonovic M, Doncheva NT, Morris JH, Bork P, Jensen LJ, and Mering CV. 2019. STRING v11: protein-protein association networks with increased coverage, supporting functional discovery in genome-wide experimental datasets. Nucleic Acids Res. 47: D607-d613. doi: 10.1093/nar/gky1131.

Tang Q, Zhang H, Kong M, Mao X, and Cao X. 2018. Hub genes and key pathways of non-small lung cancer identified using bioinformatics. Oncol Lett. 16: 2344-2354. doi:10.3892/ol.2018.8882.

Tang Y, Li M, Wang J, Pan Y, and Wu FX. 2015. CytoNCA: a cytoscape plugin for centrality analysis and evaluation of protein interaction networks. Biosystems. 127: 67-72. 
445 doi:10.1016/j.biosystems.2014.11.005.

446 Tang Z, Cai H, Wang R, and Cui Y. 2018b. Overexpression of CD300A inhibits progression of

447 NSCLC through downregulating Wnt/beta-catenin pathway. Onco Targets Ther. 11: 8875-8883.

448 doi:10.2147/ott.s185521.

449 Wang P, Yu X, and Lu J. 2014. Identification and evolution of structurally dominant nodes in 450 protein-protein interaction networks. IEEE Trans Biomed Circuits Syst. 8: 87-97. 451 doi:10.1109/tbcas.2014.2303160.

452 Wang Q, Acharya N, Liu Z, Zhou X, Cromie M, Zhu J, and Gao W. 2018. Enhanced anticancer 453 effects of Scutellaria barbata D. Don in combination with traditional Chinese medicine 454 components on non-small cell lung cancer cells. J Ethnopharmacol. 217: 140-151. 455 doi:10.1016/j.jep.2018.02.020.

456 Wen P, Chidanguro T, Shi Z, Gu H, Wang N, Wang T, Li Y, and Gao J. 2018. Identi fi cation of 457 candidate biomarkers and pathways associated with SCLC by bioinformatics analysis. Mol Med 458 Rep. 18: 1538-1550. doi:10.3892/mmr.2018.9095.

459 Xiao Y, Feng M, Ran H, Han X, and Li X. 2018. Identification of key differentially expressed 460 genes associated with nonsmall cell lung cancer by bioinformatics analyses. Mol Med Rep. 17: 461 6379-6386. doi:10.3892/mmr.2018.8726.

462 Yang G, Chen Q, Xiao J, Zhang H, Wang Z, and Lin X. 2018. Identification of genes and 463 analysis of prognostic values in nonsmoking females with non-small cell lung carcinoma by 464 bioinformatics analyses. Cancer Manag Res. 10: 4287-4295. doi:10.2147/cmar.s174409.

465 Zakaria N, Yusoff NM, Zakaria Z, Lim MN, Baharuddin PJ, Fakiruddin KS, and Yahaya B. 466 2015. Human non-small cell lung cancer expresses putative cancer stem cell markers and 467 exhibits the transcriptomic profile of multipotent cells. BMC Cancer. 15: 84. 468 doi:10.1186/s12885-015-1086-3.

469 Zhang F, Lin JD, Zuo XY, Zhuang YX, Hong CQ, Zhang GJ, Cui XJ, and Cui YK. 2017. 470 Elevated transcriptional levels of aldolase A (ALDOA) associates with cell cycle-related genes 471 in patients with NSCLC and several solid tumors. BioData Min. 10: 6. doi:10.1186/s13040-016472 0122-4.

473 Zhu Q, Sun Y, Zhou Q, He Q, and Qian H. 2018. Identification of key genes and pathways by 474 bioinformatics analysis with TCGA RNA sequencing data in hepatocellular carcinoma. Mol Clin 475 Oncol. 9: 597-606. doi:10.3892/mco.2018.1728. 
Figure 1

The identification of DEGs.

(A) The intersection of DEGs from the expression profiles GSE33532, GSE30219 and

GSE19804 were detected by Venny2.1. (B) Volcano plot of the distribution of all differentially expressed genes, including the 519 upregulated genes (red) and 166 downregulated genes (blue). The cut-off criteria: $|\log \mathrm{FC}|>1$, pvalue $<0.01$.

A

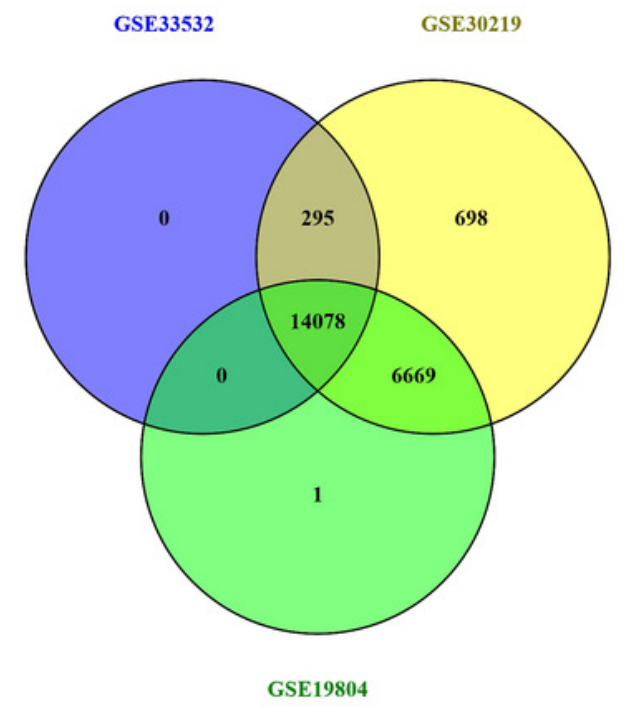

B

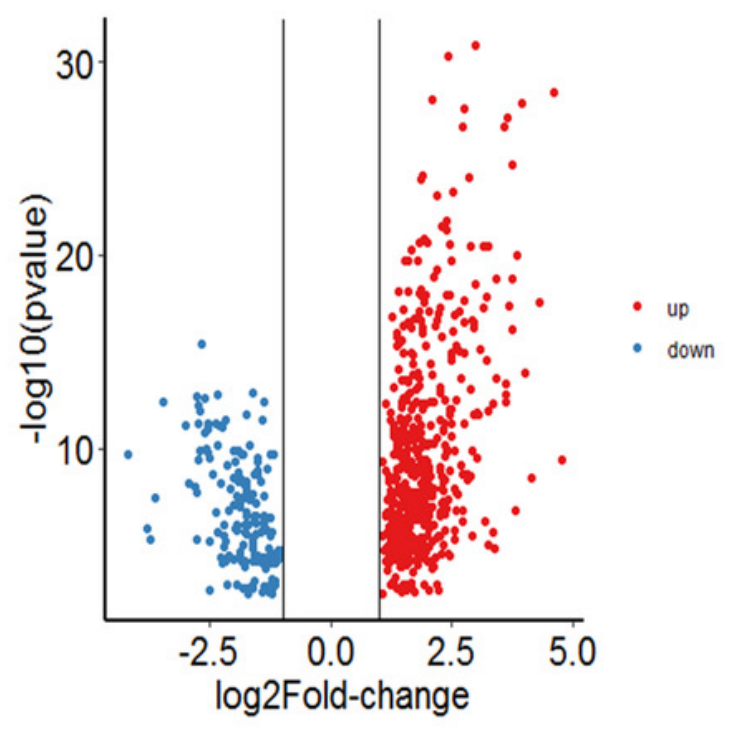


Figure 2

Functional annotation of DEGs by DAVID.

(A) GO analysis of DEGs based on their functions into three groups (molecular function, biological process and cellular component). Data showed the top 25 significant terms according to enrichment factor. (B) The KEGG pathway analysis of DEGs in NSCLC.

A

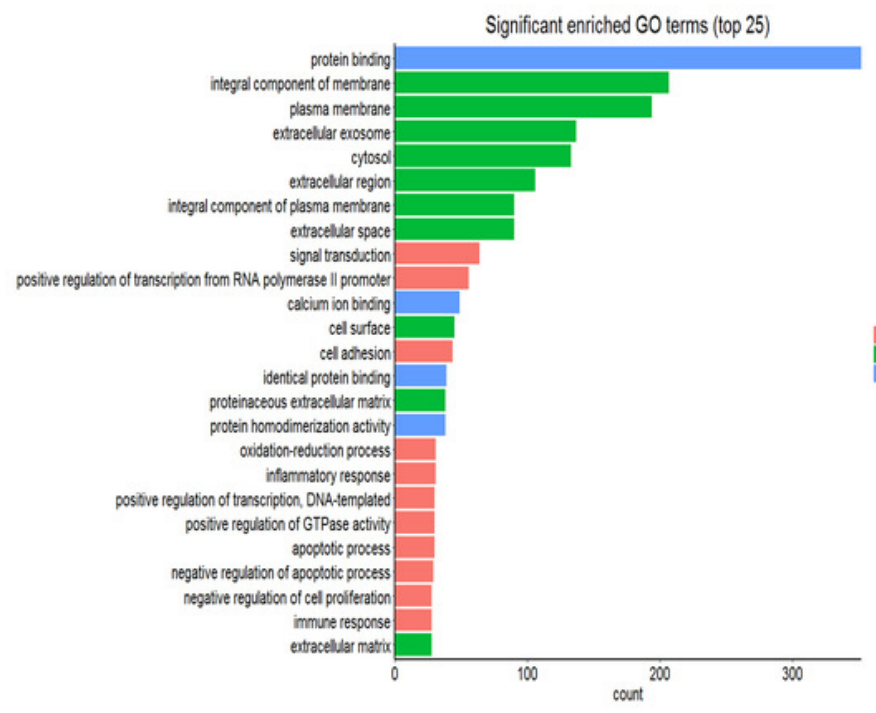

B

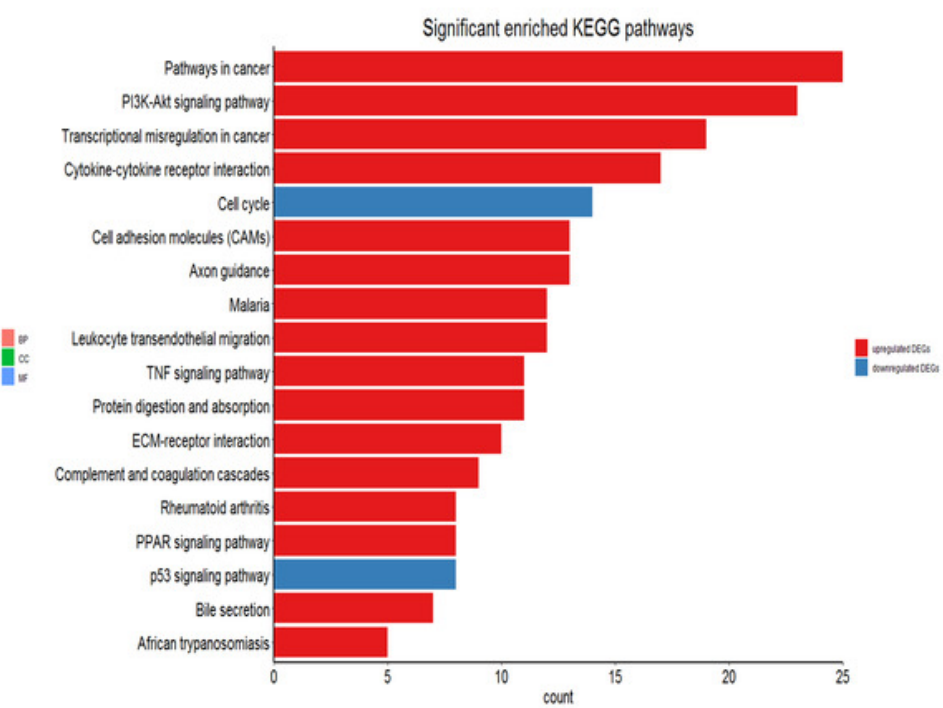




\section{Figure 3}

The centrality analysis of the NSCLC related PPI network.

(A-D) The distribution of degree centrality, betweenness centrality, closeness centrality and eigenvector centrality based in Cytoscape's plug-in network by the R language. (E) The intersection of the top $25 \%$ molecules in each centrality (degree centrality, betweenness centrality, closeness centrality and eigenvector centrality) by venny2.1.0. Results showed 16 key DEGs were chosen for further study because of their high degree, betweenness, closeness and eigenvector values.

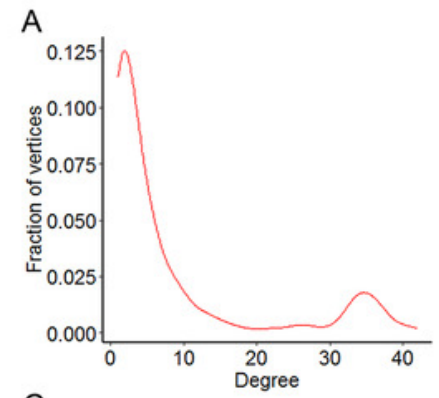

C
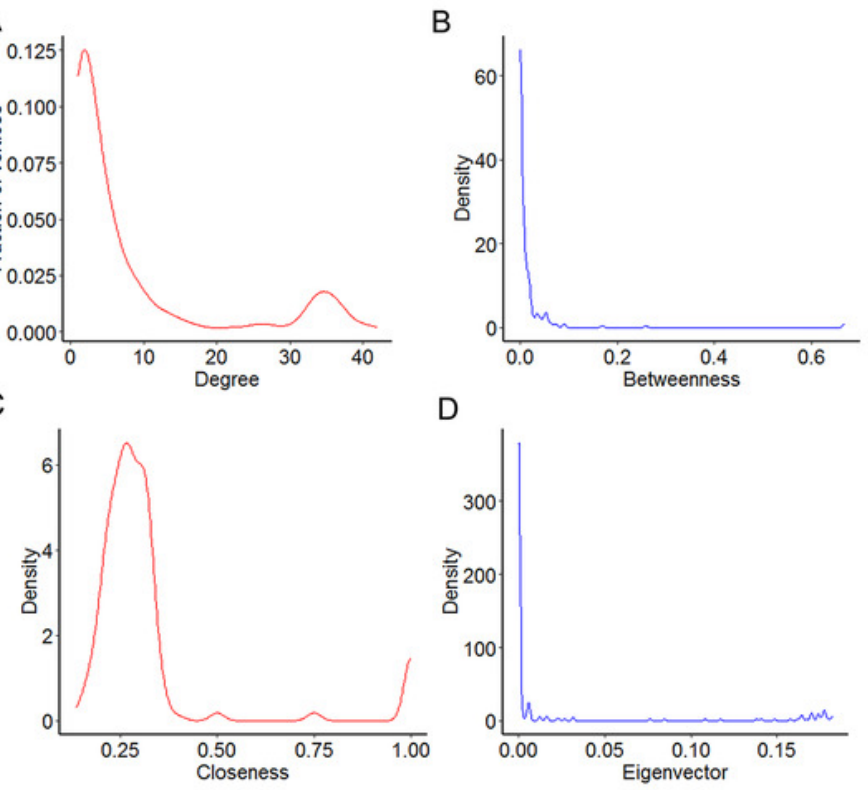

E

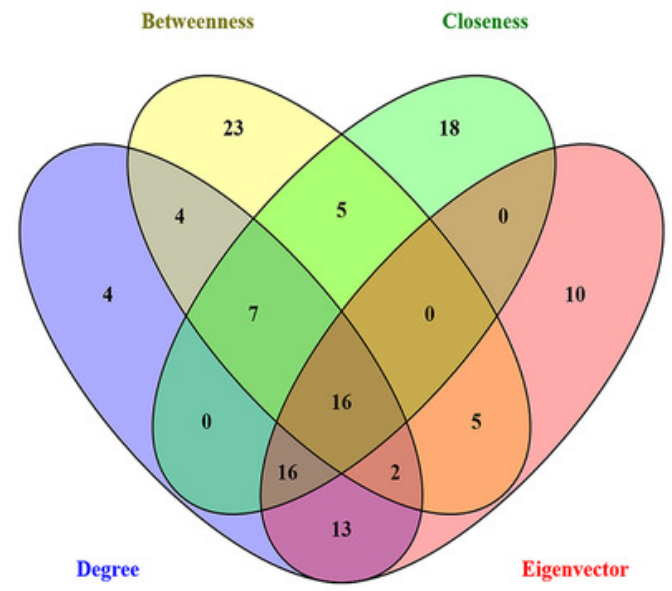




\section{Figure 4}

Survival analysis of key genes in NSCLC patients.

(A) Survival analysis of CDK1 in NSCLC patients by GEPIA. The result showed the survival of NSCLC patients with the high expressions of CDK1 was significantly worse $(p<0.01)$. (B) Survival analysis of CCNB1 in NSCLC patients by GEPIA. The result showed the survival of NSCLC patients with the high expressions of CCNB1 was significantly worse $(p<0.01)$. (C) Survival analysis of CCNA2 in NSCLC patients by GEPIA. The result showed the survival of NSCLC patients with the high expressions of CCNA2 was significantly worse $(p<0.01)$. (D) Survival analysis of BIRC5 in NSCLC patients by GEPIA. The result showed the survival of NSCLC patients with the high expressions of BIRC5 was significantly worse $(p<0.01)$. (E) Survival analysis of CCNB2 in NSCLC patients by GEPIA. The result showed the survival of NSCLC patients with the high expressions of CCNB2 was significantly worse $(p<0.01)$. (F) Survival analysis of KIAA0101 in NSCLC patients by GEPIA. The result showed the survival of NSCLC patients with the high expressions of KIAA0101 was significantly worse $(p<0.01)$. (G) Survival analysis of MELK in NSCLC patients by GEPIA. The result showed the survival of NSCLC patients with the high expressions of MELK was significantly worse $(p<0.01)$. 
A

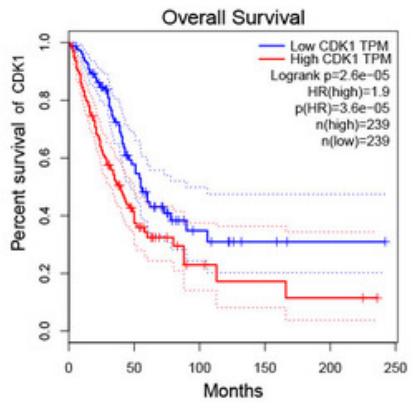

E

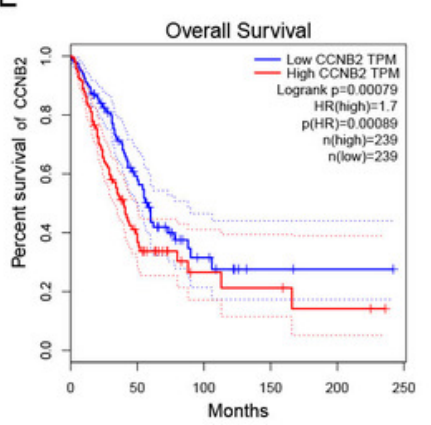

C

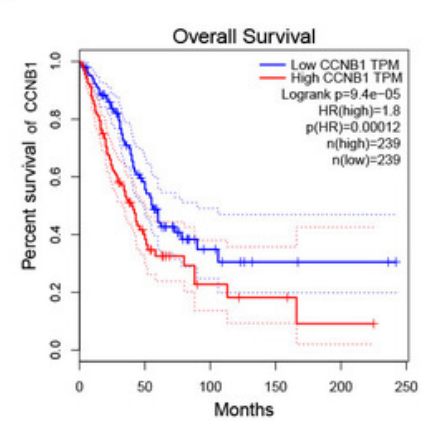

$\mathrm{F}$

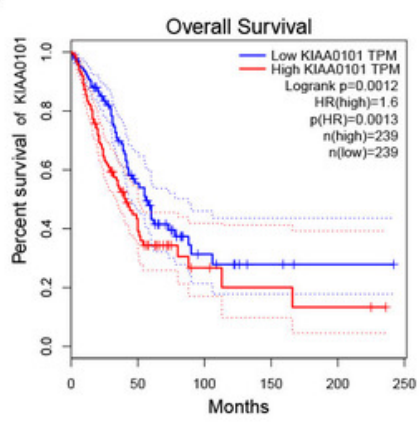

D
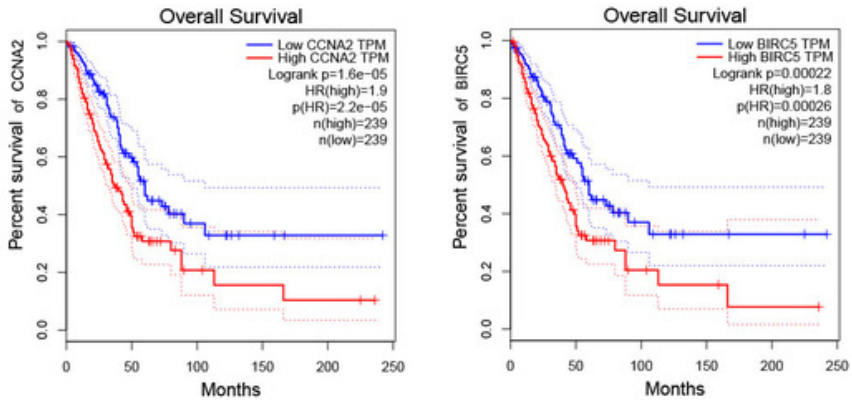

G

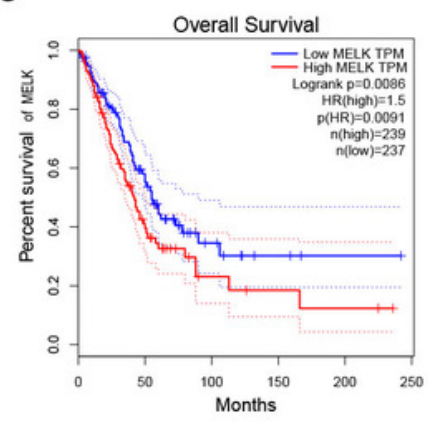




\section{Table 1 (on next page)}

The top $25 \%$ centrality parameters of 16 candidate molecules in the PPI network. 
1

\begin{tabular}{lllll}
\hline Genes name & Degree & Betweenness & Closeness & Eigenvector \\
\hline CDK1 & 42 & 0.05442045 & 0.34029851 & 0.34029851 \\
PRKCB & 10 & 0.0897726 & 0.35130971 & 0.006357714 \\
EDN1 & 26 & 0.25859441 & 0.40140845 & 0.012624903 \\
TIMP3 & 13 & 0.05680489 & 0.33878158 & 0.005104212 \\
MELK & 36 & 0.01845249 & 0.33628319 & 0.17740285 \\
SPP1 & 13 & 0.03509843 & 0.34131737 & 0.02659089 \\
PLA2G1B & 9 & 0.05464156 & 0.33878158 & 0.006098443 \\
BIRC5 & 37 & 0.0424209 & 0.36018957 & 0.1761744 \\
CCNB1 & 40 & 0.01947945 & 0.32805755 & 0.18236643 \\
KIAA0101 & 37 & 0.0555325 & 0.34285714 & 0.17740497 \\
CCNA2 & 40 & 0.02089539 & 0.32900433 & 0.18205161 \\
EZH2 & 38 & 0.09184724 & 0.36952998 & 0.15754144 \\
CCNB2 & 37 & 0.01609439 & 0.36952998 & 0.15754144 \\
LRRK2 & 22 & 0.16920811 & 0.36190476 & 0.012165083 \\
SELP & 11 & 0.05407089 & 0.34131737 & 0.006136451 \\
KLF4 & 9 & 0.05250658 & 0.34915773 & 0.016391514 \\
\hline
\end{tabular}

2 